PROCEEDINGS OF THE

AMERICAN MATHEMATICAL SOCIETY

Volume 134, Number 7, Pages 2083-2089

S 0002-9939(06)08339-0

Article electronically published on January 6, 2006

\title{
UPPER AND LOWER BOUNDS FOR THE FIRST DIRICHLET EIGENVALUE OF A TRIANGLE
}

\author{
PEDRO FREITAS \\ (Communicated by Carmen C. Chicone)
}

\begin{abstract}
We prove some new upper and lower bounds for the first Dirichlet eigenvalue of a triangle in terms of the lengths of its sides.
\end{abstract}

\section{INTRODUCTION}

With the exception of a few domains such as rectangles, balls, annuli and some triangles, the first eigenvalue $\lambda_{1}$ of the Dirichlet Laplacian of a planar domain is not known explicitly. While this is not surprising in general, we might expect that in the very special case of the triangle we would be able to say a bit more, since in this case $\lambda_{1}$ depends only on three variables (two if we allow for rescaling). Furthermore, it is known that in other spectral related issues triangles behave in a special fashion (see, for instance, $\mathrm{CD}$, where it was proven that a finite number of eigenvalues uniquely determines the triangle up to congruence). However, this is not the case, and, as far as we are aware, the only cases for which the spectrum is known explicitly are the equilateral triangle, the right isosceles triangle and the right triangle with angles $\pi / 3$ and $\pi / 6$; for attempts at more general results, see [Ga, Go.

Regarding specific estimates for $\lambda_{1}$, not much can be found in the literature either. The main result in this respect is the classical isoperimetric inequality of Pólya and Szegö, which basically states that among all triangles of equal area, the equilateral triangle minimizes the first Dirichlet eigenvalue $[\mathrm{PoSz}$. This may be written as

$$
\lambda_{1}(T) \geq \frac{4 \sqrt{3} \pi^{2}}{3 A},
$$

where $A$ denotes the area of $T$. There is also a lower bound by Brooks and Waksman BW], namely,

$$
\lambda_{1}(T) \geq \frac{(L+2 \sqrt{\pi A})^{2}}{16 A^{2}},
$$

but it is not difficult to see that this does not compare well with more general lower bounds such as the following estimate due to Makai $[\mathbf{M}]$ :

$$
\lambda_{1}(K) \geq \frac{\pi^{2} L^{2}}{16 A^{2}}
$$

Received by the editors September 9, 2004 and, in revised form, February 16, 2005.

2000 Mathematics Subject Classification. Primary 35P15; Secondary 35J05.

(C)2006 American Mathematical Society Reverts to public domain 28 years from publication 
Here $K$ is a planar convex domain and $A$ and $L$ denote its area and boundary length, respectively. In fact, this estimate may be improved upon by using the following result of Protter $[\mathrm{Pr}$ :

$$
\lambda_{1}(K) \geq \frac{\pi^{2}}{4}\left[\frac{1}{\rho^{2}}+\frac{1}{d^{2}}\right],
$$

where $\rho$ and $d$ denote the inradius and the diameter of $K$, respectively. Proceeding as in Makai's paper, we are lead to

$$
\lambda_{1}(K) \geq \frac{\pi^{2}}{4}\left[\frac{L^{2}}{4 A^{2}}+\frac{1}{d^{2}}\right] .
$$

As for upper bounds, we have the following result due to Pólya $[\mathrm{PO}]$ :

$$
\lambda_{1}(K) \leq \frac{\pi^{2} L^{2}}{4 A^{2}}
$$

valid for arbitrary doubly-connected domains; see the remark on this in $\mathrm{O}$.

Due to their simplicity, we will use these two results and (1) as comparison terms for our bounds. Also note that inequality (2) is attained asymptotically in the case of a narrow circular sector, which means that, at least in this limiting case, it should provide a good bound for triangles.

Another motivation for the present paper, is the numerical study recently carried out in $\mathrm{AF}$, which seems to point in the direction that known bounds can still be improved in the case of polygons and other planar domains. In particular, based on the numerical data gathered it is conjectured in $\mathrm{AF}]$ that

Conjecture 1. For any triangle $T$ we have

$$
\lambda_{1}(T) \leq \frac{\pi^{2} L^{2}}{9 A^{2}}
$$

While we have not been able to obtain this, we do improve on Pólya's upper bound in the particular case of triangles, and, under some conditions, on both (11) and (2) in the case of the lower bound. More precisely, we have the following.

Theorem 1. Let $T$ be a triangle with sides of lengths $\ell_{1} \leq \ell_{2} \leq \ell_{3}$. Then

$$
\pi^{2}\left(\frac{4}{\ell_{3}^{2}}+\frac{\ell_{3}^{2}}{4 A^{2}}\right) \leq \lambda_{1}(T) \leq \frac{\pi^{2}}{3 A^{2}}\left(\ell_{1}^{2}+\ell_{2}^{2}+\ell_{3}^{2}\right)
$$

where $A$ denotes the area of $T$.

Remark 1.1. In both cases equality holds for the equilateral triangle and this is the only instance where the two bounds agree; see the last section. However, the lower bound does give values lower than this value for a certain range of $\ell_{3}$ and $A$.

Remark 1.2. The area $A$ may, of course, be written in terms of the lengths of the sides of $T$ only, by means of Heron's formula, and so the bounds given above depend in fact only on $\ell_{1}, \ell_{2}$ and $\ell_{3}$.

We prove the lower bound in the next section, the upper bound in Section 3 , and in the last section discuss the results obtained and compare them with the bounds (11), (2) and (3). 


\section{PROOF OF THE LOWER BOUND}

The idea for the proof of the lower bound is to build a test function for the second eigenvalue of a flat cylinder by using the first eigenfunction of the triangle in question. In order to do this, and given a triangle $T$ with sides $\ell_{1} \leq \ell_{2} \leq \ell_{3}$, we begin by considering the rectangle $R$ with sides $\ell_{3}$ and $h_{3}$, where $h_{3}$ is the height of $T$ with respect to $\ell_{3}$. If we denote by $T_{1}$ and $T_{2}$ the two right triangles that $T$ is divided into by $h_{3}$, then the rectangle $R$ will contain precisely two copies of each $T_{1}$ and $T_{2}$. We shall denote by $T_{3}$ and $T_{4}$ the two triangles which are isometric to $T_{1}$ and $T_{2}$, respectively; see Figure 1 .

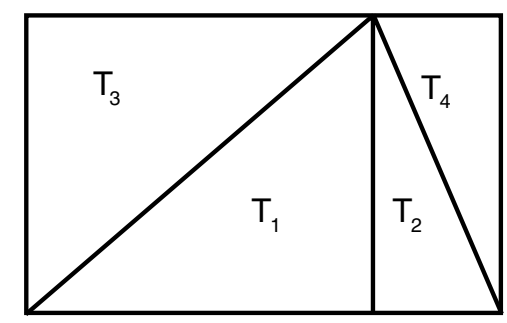

Figure 1. Decomposition of $\mathrm{C}$ into four triangles

We shall now identify the two shortest sides of $R$ with each other, and consider the Dirichlet eigenvalue problem on the flat cylinder $C$ obtained in this way. We have that

$$
\lambda_{1}(C)=\frac{\pi^{2}}{h_{3}^{2}} \quad \text { with eigenfunction } \quad u_{1}(x, y)=\sin \left(\frac{\pi y}{h_{3}}\right),
$$

while the second eigenvalue is given by

$$
\lambda_{2}(C)=\min \left\{\frac{4 \pi^{2}}{\ell_{3}^{2}}+\frac{\pi^{2}}{h_{3}^{2}}, \frac{4 \pi^{2}}{h_{3}^{2}}\right\}=\frac{4 \pi^{2}}{\ell_{3}^{2}}+\frac{\pi^{2}}{h_{3}^{2}}=\pi^{2}\left(\frac{4}{\ell_{3}^{2}}+\frac{\ell_{3}^{2}}{4 A^{2}}\right),
$$

since $\ell_{3}$ is the largest side, and thus $h_{3}$ is always less than or equal to $\sqrt{3} \ell_{3} / 2$, which is attained for the equilateral triangle. In this case we have a double eigenvalue whose corresponding eigenfunctions are given by

$$
u_{2}(x, y)=\sin \left(\frac{\pi y}{h_{3}}\right)\left[c_{1} \cos \left(\frac{2 \pi x}{\ell_{3}}\right)+c_{2} \sin \left(\frac{2 \pi x}{\ell_{3}}\right)\right] .
$$

On the other hand, we have that

$$
\lambda_{2}(C)=\inf _{\varphi \in X} \frac{\int_{C}|\nabla \varphi|^{2} d x d y}{\int_{C} \varphi^{2} d x d y},
$$

where $X$ is the set of continuous functions in $C$, whose gradient belongs to $\mathcal{L}^{2}(C)$, take zero values on $\partial C$, do not vanish identically and, furthermore, are orthogonal to $u_{1}$ on $C$. We shall now construct a test function for the second eigenvalue of $C$ based on the first eigenfunction of $T$ which we shall denote by $v$. Let

$$
\varphi= \begin{cases}v, & \text { on } T_{1} \cup T_{2} \\ v_{13}, & \text { on } T_{3} \\ v_{24}, & \text { on } T_{4}\end{cases}
$$


where $v_{13}$ is the function obtained from $v$ by rotating the triangle $T_{1}$ around the middle point of its largest side until it coincides with $T_{3}$, and then changing its sign. In a similar way, one obtains $v_{24}$ from $v$ on $T_{2}$.

From the expression for $u_{1}$ and the construction of $\varphi$ we see that

$$
\int_{C} \varphi u_{1} d x d y=\int_{T_{1}} v u_{1} d x d y+\int_{T_{2}} v u_{1} d x d y+\int_{T_{3}} v_{13} u_{1} d x d y+\int_{T_{4}} v_{24} u_{1} d x d y
$$

vanishes, so that $\varphi$ may be used as a test function for the second eigenvalue of $C$. Replacing this test function in the Rayleigh quotient (4) we obtain that

$$
\lambda_{2}(C) \leq \frac{\int_{T_{1} \cup T_{2}}|\nabla v|^{2} d x d y+\int_{T_{3}}\left|\nabla v_{13}\right|^{2} d x d y+\int_{T_{4}}\left|\nabla v_{24}\right|^{2} d x d y}{\int_{T_{1} \cup T_{2}} v^{2} d x d y+\int_{T_{3}} v_{13}^{2} d x d y+\int_{T_{4}} v_{24}^{2} d x d y}=\lambda_{1}(T),
$$

which gives the desired result.

\section{Proof OF THE UPPER Bound}

In order to obtain the upper bound, we shall now use a test function based on the first eigenfunction of the equilateral triangle. For simplicity, we shall begin by proving the result for a triangle $T^{\prime}$ with vertices at the origin, $(1,0)$ and $(a, b) \in$ $\mathbb{R} \times \mathbb{R}^{+}$, and then rescale to obtain the upper bound given in the theorem. We will not assume any restrictions on the relative lengths of the sides, since in this case this is irrelevant.

Consider the first eigenfunction of the equilateral triangle with vertices at the origin, $(1,0)$ and $(1 / 2, \sqrt{3} / 2)$, that is,

$$
f(x, y)=\sin \left(\frac{4 \pi y}{\sqrt{3}}\right)-\sin \left[2 \pi\left(x+\frac{y}{\sqrt{3}}\right)\right]+\sin \left[2 \pi\left(x-\frac{y}{\sqrt{3}}\right)\right] .
$$

We will now perform a linear transformation in order to be able to use this as a test function for the first eigenvalue of $T^{\prime}$. Thus, let

$$
\begin{aligned}
\varphi(x, y) & =f\left(x-\frac{a-1 / 2}{b} y, \frac{\sqrt{3}}{2 b} y\right) \\
& =\sin \left(2 \pi \frac{y}{b}\right)-\sin \left[2 \pi \frac{b x+(1-a) y}{b}\right]+\sin \left[2 \pi\left(x-\frac{a y}{b}\right)\right] .
\end{aligned}
$$

Clearly, $\varphi$ satisfies the boundary conditions on $T^{\prime}$, and, after some lengthy but straightforward computations, we obtain

$$
\int_{T^{\prime}}|\nabla \varphi(x, y)|^{2} d x d y=\frac{2 \pi^{2}}{b}\left[1+(a-1) a+b^{2}\right] \text { and } \int_{T^{\prime}} \varphi(x, y)^{2} d x d y=\frac{3 b}{4} .
$$

Plugging this into the Rayleigh quotient yields

$$
\lambda_{1}\left(T^{\prime}\right) \leq \frac{8 \pi^{2}}{3 b^{2}}\left[1+(a-1) a+b^{2}\right] .
$$


If we now rescale $T^{\prime}$ by multiplication by $\ell_{1}$, we obtain a triangle, say $T$, with vertices at the origin, $\left(\ell_{1}, 0\right)$ and $(\alpha, \beta)=\ell_{1}(a, b)$. With this notation, we have

$$
\begin{aligned}
\lambda_{1}(T) & \leq \frac{8 \pi^{2}}{3 b^{2} \ell_{1}^{2}}\left[1+(a-1) a+b^{2}\right] \\
& =\frac{8 \pi^{2}}{3 \beta^{2}}\left[1+\left(\frac{\alpha}{\ell_{1}}-1\right) \frac{\alpha}{\ell_{1}}+\frac{\beta^{2}}{\ell_{1}^{2}}\right] \\
& =\frac{\pi^{2}}{3 A^{2}}\left(\ell_{1}^{2}+\ell_{2}^{2}+\ell_{3}^{2}\right),
\end{aligned}
$$

where the last indentity follows from the relations

$$
\ell_{2}=\sqrt{\alpha^{2}+\beta^{2}} \text { and } \ell_{3}=\sqrt{\left(\ell_{1}-\alpha\right)^{2}+\beta^{2}} .
$$

\section{Discussion}

We begin by proving the assertion made in Remark 1.1 that the upper and lower bounds agree only in the case of the equilateral triangle. To see this, equate the two bounds to obtain that

$$
48 A^{2}=4\left(\ell_{1}^{2}+\ell_{2}^{2}\right) \ell_{3}^{2}+\ell_{3}^{4} .
$$

We have that if $T$ is not equilateral, then $h_{3}<\sqrt{3} \ell_{3} / 2$ and thus $\ell_{3}^{2}>4 \sqrt{3} A / 3$, giving, upon substitution in (5), that

$$
h_{3}^{2}>\frac{3}{8}\left(\ell_{1}^{2}+\ell_{2}^{2}\right) .
$$

On the other hand, and denoting by $\tau$ and $\ell_{3}-\tau$ the lengths of the line segments that the longest side of $T$ is divided into by the corresponding height, we have that

$$
\begin{aligned}
\ell_{1}^{2}+\ell_{2}^{2} & =2 h_{3}^{2}+\tau^{2}+\left(\ell_{3}-\tau\right)^{2} \\
& \geq 2 h_{3}^{2}+\frac{1}{2} \ell_{3}^{2} \\
& \geq 2 h_{3}^{2}+\frac{1}{4}\left(\ell_{1}^{2}+\ell_{2}^{2}\right),
\end{aligned}
$$

yielding

$$
\ell_{1}^{2}+\ell_{2}^{2} \geq \frac{8}{3} h_{3}^{2}
$$

which contradicts (6).

Now we compare the bounds obtained with those mentioned in the Introduction, and we begin by comparing our lower bound with that of Pólya and Szegö's. It is easy to check that we will have

$$
\pi^{2}\left(\frac{4}{\ell_{3}^{2}}+\frac{\ell_{3}^{2}}{4 A^{2}}\right)>\frac{4 \sqrt{3} \pi^{2}}{3 A} \quad \text { if and only if } \quad h_{3}<\frac{\sqrt{3}}{6} \ell_{3},
$$

where, as before, $h_{3}$ denotes the height with respect to the longest side $\ell_{3}$. This was to be expected, since their bound depends only on the area, and thus cannot be very good away from the equilateral triangle.

The comparison with Makai's lower bound is not as straightforward, but it is still possible to see that our bound does improve on (2) for a class of triangles. Due to the above comparison with Pólya and Szegö's bound, we will concentrate on the case of small $h_{3}$. 
We want to check when it is that

$$
\frac{4}{\ell_{3}^{2}}+\frac{\ell_{3}^{2}}{4 A^{2}}>\frac{L^{2}}{16 A^{2}}+\frac{1}{4 \ell_{3}^{2}}
$$

holds. Consider, without loss of generality, the triangle with two vertices at $(0,0)$ and $\left(\ell_{3}, 0\right)$ and write the $x$-coordinate of the remaining vertex as $\alpha \ell_{3}$ for some $\alpha$ on $[0,1]$. Then, the above condition is equivalent to

$$
F\left(\alpha, h_{3}, \ell_{3}\right)=15 h_{3}^{2}+4 \ell_{3}^{2}-\left[\sqrt{\alpha^{2} \ell_{3}^{2}+h_{3}^{2}}+\sqrt{(1-\alpha)^{2} \ell_{3}^{2}+h_{3}^{2}}+\ell_{3}\right]^{2}>0 .
$$

If we expand $F$ in $h_{3}$ around zero we obtain

$$
F\left(\alpha, h_{3}, \ell_{3}\right)=\left[15-\frac{2}{\alpha(1-\alpha)}\right] h_{3}^{2}+o\left(h_{3}^{4}\right),
$$

showing that $F$ will be positive for sufficiently small values of $h_{3}$, provided $\alpha$ belongs to $((15-\sqrt{105}) / 30,(15+\sqrt{105}) / 30)$.

Finally, we compare our upper bound with (3), namely, we want to know when

$$
G\left(\ell_{1}, \ell_{2}, \ell_{3}\right)=\left(\ell_{1}+\ell_{2}+\ell_{3}\right)^{2}-\frac{4}{3}\left(\ell_{1}^{2}+\ell_{2}^{2}+\ell_{3}^{2}\right)>0 .
$$

Clearly, the above inequality will not hold in general. However, if we take into account the triangle inequality and the fact that the side lengths are nonnegative, it is a simple exercise in constrained optimization to see that the minimum value of the function $G$, subject to these restrictions, occurs at the origin.

We finish by pointing out that in the last case, instead of using a test function based on the first eigenfunction alone, it is possible to use a linear combination of the first $m$ eigenfunctions. However, the number of computations necessary to proceed in this fashion grows very quickly, making the process rather cumbersome.

\section{REFERENCES}

[AF] P. Antunes and P. Freitas, New bounds for the principal Dirichlet eigenvalue of planar regions, preprint.

[BW] R. Brooks and P. Waksman, The first eigenvalue of a scalene triangle, Proc. Amer. Math. Soc. 100 (1987), 175-182. MR0883424 (88f:58147)

[CD] P.-K. Chang and D. Deturck, On hearing the shape of a triangle, Proc. Amer. Math. Soc. 105 (1989), 1033-1038. MR0953738(89h:58194)

[Ga] M. Gaudin, Vers le spectre du triangle?, J. Physique 48 (1987), 1633-1650. MR0923667 (89e:58113)

[Go] T. Gorin, Generic spectral properties of right triangle billiards, J. Phys. A 34 (2001), 8281-8295. MR1873184 (2002j:82006)

[M] E. Makai, On the principal frequency of a membrane and the torsional rigidity of a beam, pp. 227-231 in Studies in mathematical analysis and related topics, Essays in honor of George Pólya, Stanford Univ. Press, Stanford 1962. MR0167004 (29:4277)

[O] R. Osserman, A note on Hayman's theorem on the bass note of a drum, Comment. Math. Helvetici 52 (1977), 545-555. MR0459099 (56:17297)

[Po] G. Pólya, Two more inequalities between physical and geometrical quantities, J. Indian Math. Soc. (N.S.)24 (1960), 413-419. MR0133059 (24:A2895) 
[PoSz] G. Pólya and G. Szegö, Isoperimetric inequalities in mathematical physics, Annals of Mathematical Studies 27, Princeton University Press, Princeton, 1951. MR0043486 (13:270d)

[Pr] M. H. Protter, A lower bound for the fundamental frequency of a convex region, Proc. Amer. Math. Soc. 81 (1981), 65-70. MR0589137 (82b:35113)

Departamento de Matemática, Instituto Superior Técnico, Av. Rovisco Pais, 1049001 Lisboa, Portugal

E-mail address: pfreitas@math.ist.utl.pt

Current address: Faculdade de Motricidade Humana and Mathematical Physics Group of the University of Lisbon, Complexo Interdisciplinar, Av. Prof. Gama Pinto 2, P-1649-003 Lisboa, Portugal

E-mail address: freitas@cii.fc.ul.pt 